\title{
A Ferroelastic Film at the Edge of Chaos
}

\section{Period doubling - a behavior seen in systems that are nearing a chaotic regime-shows up in the microstructure of a strain-textured material.}

\section{by Pol Lloveras*}

$\mathrm{F}$ erroelastic materials are perhaps best known for their shape memory effect in which they return to their original shape after being molded. The signature of a ferroelastic material is that its crystal structure will distort when the material is stretched or changed in temperature by a sufficient amount. This structural transition often leads to the formation of "twins" - regions where the structure is deformed (strained) by the same amount but in different orientations. Now, an intriguing similarity between the behavior of twin patterns and systems that are nearing a chaotic regime has been found by Arnoud Everhardt and Silvia Damerio of the University of Groningen, Netherlands, and collaborators [1]. The team imaged a twin pattern in a ferroelastic film and observed that the pattern's periodicity doubled repeatedly as the film was warmed to progressively
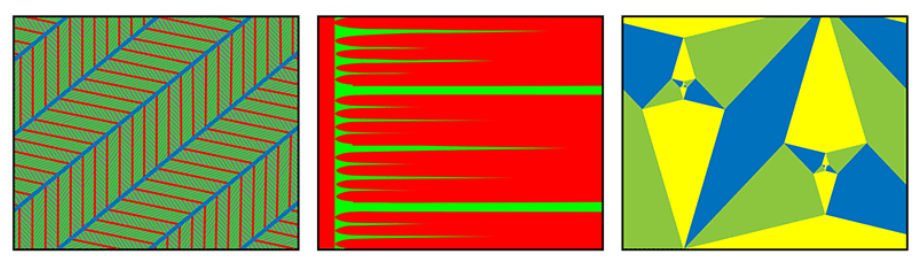

Figure 1: Ferroelastics can have intricate twin patterns, such as twins within twins (left), branched twins (center), and star-shaped twins (right). In the center and right images, the colors represent twins of differing orientation; in the left image, the lines denote the boundaries between twins. (APS/Alan Stonebraker)

\footnotetext{
* Department of Physics, Universitat Politècnica de Catalunya and Barcelona Research Center in Multiscale Science and Engineering, Barcelona, Catalonia, Spain
}

higher temperatures. Other experiments have hinted at a connection between ferroelastics and nearly chaotic systems. But this is the first evidence of a period-doubling "cascade," which strengthens the connection and may encourage more physicists to apply concepts from chaos to ferroelastics.

A simple example of a ferroelastic is a material whose crystal structure, on cooling, switches from cubic to tetragonal (a cube elongated in one direction). The low-temperature tetragonal structure nucleates and grows within the hightemperature cubic structure. The lattice misfit at the phase boundaries costs elastic energy and could eventually lead to the break up of the lattice. To maintain the lattice integrity and release the elastic energy, the lattice relaxes through a process called self-accommodation [2, 3], forming regions (twins) with the same energy and crystal symmetry but with the distortion oriented in different directions. (For example, in the above case, one of the twin regions might have tetragons orientated horizontally, while the other region would have vertically oriented tetragons.) Long-range elastic interactions cause twins to span across the entire material, forming intricate patterns that range from simple parallel bands, to herringbone, star-shaped, and spiral patterns [2-4]. The characteristic lengths over which these patterns repeat are often determined by the size of larger superstructures, such as the distance between phase boundaries, the thickness of the material, or superdomains [3].

The rules for pattern formation extend from the atomic to the macroscopic scale [2], leading to twin patterns with multiscale behavior that is reminiscent of nearly chaotic systems. For example, twin configurations can be "hierarchical," such as twins within twins [5] or single twins that branch into multiple twins [6] (Fig. 1). Another evocative behavior is the dynamic increase in the number of twins when approaching the ferroelastic phase transition on heating [7]. Further, the motion of twin interfaces can emit acoustic waves whose amplitudes, energies, and durations are scale-free; that is, they don't exhibit any characteristic length scale [8]. Like chaotic systems - whose behavior appears random but is in fact deterministic - ferroelastics can also be very sensitive to initial conditions. For example, the twin configuration may depend on the material's size, its thermal or external-field history, or the density of its defects [2].

The cascade behavior observed by Everhardt, Damerio, et 

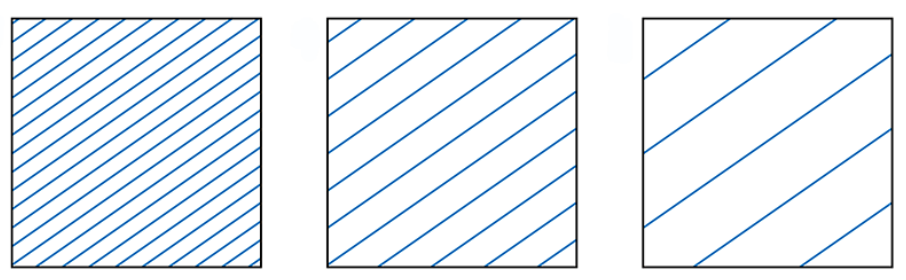

Figure 2: Everhardt, Damerio, et al. studied the ferroelastic $\mathrm{BaTiO}_{3}$ and observed a twin pattern that doubled in periodicity as the material was warmed and halved in periodicity as it was cooled [1]. This type of period-doubling cascade is sometimes seen in systems that are nearing a chaotic regime. (APS/Alan

Stonebraker)

al. helps to cement the connection between ferroelastics and chaotic systems. The team focused on the ferroelastic barium titanate $\left(\mathrm{BaTiO}_{3}\right)$, which is also a prototypical ferroelectric used in electronic and optical devices. They grew a film of $\mathrm{BaTiO}_{3}$ on a substrate, whose crystal structure imposed a small strain on the ferroelastic film [1]. When cooled below $50^{\circ} \mathrm{C}, \mathrm{BaTiO}_{3}$ transitions from a "pseudotetragonal" to "pseudo-orthorhombic" crystal structure, forming twins of the latter phase in the process. To probe the crystal structure, the team used piezoresponse force microscopy, which revealed band-like configurations of twins. To the researchers' surprise, the width of the bands doubled (period doubling) 3 times-from $100 \mathrm{~nm}$ to $200 \mathrm{~nm}$, then to $400 \mathrm{~nm}$, and finally to $800 \mathrm{~nm}$ - as the temperature was progressively increased. The process appeared to be reversible since, on cooling, the authors observed period halving, in which the bandwidth dropped from $500 \mathrm{~nm}$ to $250 \mathrm{~nm}$ and then to about $125 \mathrm{~nm}$.

Period-doubling cascades are commonly found in systems that are close to a chaotic regime, such as biological populations, flowing liquids, or economies. However, in these cases, the period doubling is typically a function of time, not temperature. Also, the doubling applies to the number of steady states achievable by the system, not a spatial parameter (like the modulation of strain). Still, the similarities are striking enough that the researchers claim their film is also "on the edge of chaos." Similar arguments have been made about other ferroics (ferromagnets, ferroelectrics [5]), suggesting that the entire class of materials could be described in a more general theoretical framework. In their case, Everhardt, Damerio, et al. ascribe the period-halving cascade to an additional self-accommodation process that is driven by a rise in equilibrium strain as the temperature drops.

Are ferroelastic systems restricted to the edge of chaos?
Researchers have seen chaotic-like behavior in (nonferroelastic) amorphous materials, frustrated systems, and spin glasses-all systems where long-range crystalline order is absent [9]. Perhaps similar behavior could be found in ferroic materials that are also lacking in long-range crystalline order, such as strain glasses, where spatially-disordered, strained nanoregions replace long-range twinning [10]. Researchers might then establish a fundamental framework for glassy ferroelastic systems that borrows ideas from chaos theory. For example, the material's sensitivity to a parameter (like composition) could be connected to a chaotic system's sensitivity to initial conditions.

The observed period doubling may have implications for applications in ferroic materials. Ferroic twin patterns and the way they evolve in an external field, with temperature or under some other stimulus, can affect properties with technological value. Examples include the shape memory effect, superelasticity [2], thermal stability, and remanent magnetization and polarization [5]. The domain boundaries can also be useful, acting as paths for enhanced charge transport or electric polarity [11]. Controlling these functionalities requires a deep understanding of the microstructure behavior, and the study by Everhardt, Damerio, et al. is an important step in this direction.

This research is published in Physical Review Letters.

\section{REFERENCES}

[1] A. S. Everhardt, S. Damerio, J. A. Zorn, S. Zhou, N. Domingo, G. Catalan, E. K. H. Salje, L.-Q. Chen, and B. Noheda, "Periodicity-doubling cascades: Direct observation in ferroelastic materials," Phys. Rev. Lett. 123, 087603 (2019).

[2] K. Bhattacharya, Microstructure of martensite why it forms and how it gives rise to the shape memory effect (Oxford University Press, Oxford, 2003).

[3] M. Porta, T. Castán, P. Lloveras, T. Lookman, A. Saxena, and S. R. Shenoy, "Interfaces in ferroelastics: Fringing fields, microstructure, and size and shape effects," Phys. Rev. B 79, 214117 (2009).

[4] C. Manolikas and S. Amelinckx, "Phase transitions in ferroelastic lead orthovanadate as observed by means of electron microscopy and electron diffraction. I. Static observations," Phys. Stat. Sol. A 60, 607 (1980).

[5] N. T. Tsou, P. R. Potnis, and J. E. Huber, "Classification of laminate domain patterns in ferroelectrics," Phys. Rev. B 83, 184120 (2011).

[6] Y. Ni, D. Yang, and L. He, "Spontaneous wrinkle branching by gradient stiffness," Phys. Rev. E 86, 031604 (2012).

[7] X. R. Huang, S. S. Jiang, X. B. Hu, X. Y. Xu, W. Zeng, D. Feng, and J. Y. Wang, "One-dimensional behavior of ferroelastic domain arrays in neodymium pentaphosphate," Phys. Rev. B 52, 9932 (1995).

[8] E. K. H. Salje, D. Xue, X. Ding, K. A. Dahmen, and J. F. Scott, "Ferroelectric switching and scale invariant avalanches in $\mathrm{BaTiO}_{3}$," Phys. Rev. Materials 3, 014415 (2019). 
[9] I. Regev, J. Weber, C. Reichhardt, K. A. Dahmen, and T. Lookman, "Reversibility and criticality in amorphous solids," Nat. Commun. 6, 8805 (2015); M. Kawamura, R. Tokunaga, and M. Okada, "Low-dimensional chaos induced by frustration in a non-monotonic system," Eur. Phys. Lett. 62, 657 (2003); F. Ritort, "Static chaos and scaling behavior in the spin-glass phase," Phys. Rev. B 50, 6844 (1994).

[10] S. Sarkar, X. Ren, and K. Otsuka, "Evidence for strain glass in the ferroelastic-martensitic system $\mathrm{Ti}_{50-x} \mathrm{Ni}_{50+x}$," Phys. Rev. Lett. 95, 205702 (2005).

[11] D. D. Viehland and E. K.H. Salje, "Domain boundarydominated systems: Adaptive structures and functional twin boundaries," Adv. Phys. 63, 267 (2014).

10.1103/Physics.12.94 